\title{
INDEX TO VOLUME 59
}

\author{
INDEX OF ABSTRACTS
}

Subjects:

Algebra and Theory of Numbers, 40, 53, 66, 75, 148, 232, 338, 373, 388, 449, 522.

Analysis, 42, 57, 66, 78, 156, 236, 344, 377, 391, 452, 531.

Applied Mathematics, 47, 60, 70, 82, 167, 243, 360, 383, 395, 455, 549.

Geometry, 48, 61, 71, 83, 171, 245, 365, 384, 395, 456, 554.

Logic and Foundations, 49, 72, 84, 174, 245, 365, 396, 556.

Statistics and Probability, 50, 84, 175, 246, 366, 385, 397, 456, 558.

Topology, 50, 61, 73, 176, 247, 366, 386, 397, 457, 559.

Authors:

Abraham, C. E., 348; Abramowitz, M., 360, 360; Adams, E., 84; Adamson, I. T. A. C., 338, 339; Agmon, S., 344; Albert, A. A., 522; Amitsur, S. A., 522; Anderson, A. G., 246; Anderson, R. D., 176, 247, 366, 559; Andrushkiw, J., 42, 236, 344; Antosiewicz, H. A., 344, 531; Apostol, T. M., 449; Arens, R., 532; Armstrong, J. W., 377; Aronszajn, N., 167, 378, 532; Arsove, M. G., 78, 532; Auslander, L., 554; Austin, D. G., 378.

Bade, W. G., 156; Bagemihl, F., 452, 532; Ball, B. J., 176, 457; Barsotti, I., 171; Bartels, R. C. F., 559; Bartle, R. G., 156, 560; Baum, J. D., 366; Baum, W. R., 560; Beckenbach, E. F., 79; Becker, H. W., 148, 522; Bell, P. O., 71; Berg, P. W., 360; Bernardi, S. D., 157; Bernholtz, B., 79; Bers, L., 360; Bing, K., 396; Bing, R. H., 177; Blackman, J., 345; Block, H. D., 157, 167; Blum, E. K., 236, 345; Blumenthal, L. M., 555; Bohun-Chudyniv, V., 232, 523; Bonsall, F. F., 157; Boothby, W. M., 555; Borel, A., 457; Bott, R., 177, 386; Botts, T. A., 560; Boyd, E., 533; Brauer, A. T., 53, 523, 523; Brauer, G. U., 533; Bremermann, H. J., 157; Brenner, J. L., 388; Browder, F. E., 42, 237, 345, 533, 534; Brown, L., 378; Brown, M., 367; Brownell, F. H., 391; Bruck, R. H., 148, 149; Brunk, H. D., 158; Buck, R. C., 66, 66; Burgess, C. E., 397; Butzer, P. L., 158, 237, 345; Byers, G. C., 524.

Calabi, E., 171; Calderon, A. P., 452, 534; Calloway, J. M., 149; Cameron, R. H., 378; Capel, C. E., 61; Cargal, B., 379: Carlitz, L., 40, 40, 53, 53, 53, 149, 150, 150, 233, 233, 233, 339, 339, 339, 340, 340, 340, 340, 524, 524, 524; Cesari, L., 67, 158, 534; Chamberlin, R. E., 177; Charnes, A., 455; Chehata, C. G., 40, 373, 373; Chen, K. T., 340; Chen, Y. W., 67, 168; Chiarulli, P., 361; Chowla, S., 340, 346; Chrestenson, H. E., 391, 392; Chung, K. L., 246, 558; Churchill, R. V., 168; Civin, P., 392; Clarke, F. M., 524; Clarkson, M. H., 346; Clement, P. A., 392; Cobbe, A. P., 40; Coburn, N., 168, 383, 550; Cohen, E., 150, 150, 233, 233; Cohen, H., 367; Cohn, H., 151, 341, 374; Collins, H. S., 42; Conrad, P. F., 151; Conte, S. D., 67; Cooke, K. L., 392; Court, N. A., 171; Cowling, V. F., 43; Coxeter, H. S. M., 365; Curtis, C. W., 151, 151, 525; Curtis, M. L., 367, 367.

Danskin, J. M., 159; Darsow, W. F., 237; Davis, A. C., 75; Davis, M. D., 49; Davis, P., 531; Davis, R. B., 535; Davis, R. M., 346; Dean, B. V., 53; DeBreu, G., 152; DeCicco, J., 172, 173, 384, 385, 555; Dekker, J. C. E., 72, 72, 72; Demers, M. R., 535; Derry, D., 395; DeVogelaere, R. J., 384; Diaz, J. B., 43, 346; Dickinson, A. B., 50; Dilworth, R. P., 525; DiPrima, R. C., 361; Doberly, V. J., 234; Dolph, C. L., 70, 550; Donoghue, W. F., 535; Douglas, Jim, Jr., 159; Douglis, A., 347; Downing, A. C., Jr., 549; Doyle, T. C., 47; Dressel, F. G., 350; Dubois, D. W., 341; Duffin, R. J., 347; Dunford, N., 159; Dvoretzky, A., 84, 85; Dyer, E., 177.

Eberlein, W. F., 347, 374, 535; Edrei, A., 347; Ehlers, F. E., 455; Elliott, J., 43; Ellis, D. O., 61, 152, 178, 525; Ellis, H. W., 536; Ellis, J. W., 61; Ellis, R., 368; Epstein, Bernard, 238; Erdös, P., 393, 452, 536; Estill, M. E., 62, 368; Ettlinger, H. J., 346, 348; Evans, G. W., II, 82; Evans, R. L., 379, 550; Evans, T., 54, 341.

Fadell, E. R., 560; Fagen, R. E., 378; Faulkner, F., 169; Federer, H., 238, 348, 
348; Feidner, J. B., 561; Feller, W., 44, 44; Fettis, H. E., 169; Ficken, F. A., 349, 368; Finn, R., 536; Fischer, I., 525; Flanders, H., 76, 172; Fleishman, B. A., 349; Floyd, E. E., 368, 368, 386, 560; Forsythe, G. E., 169, 393, 551; Fort, M. K., Jr., 386; Foster, A. L., 450, 450, 530; Fox, C., 536; Fox, J. L., 363; Fox, R. H., 340; Fraissé, R., 341, 389; Frame, J. S., 525; Frank, E., 537; Friedman, B., 537; Fullerton, R. E., $68,537$.

Gaffney, M. P., Jr., 349; Gál, I. S., 561, 561; Gale, D., 361, 556; Garabedian, P. R., 160, 538; García, M., 450; Gehring, F. W., 350; Gelbaum, B. R., 526; Gergen, J. J., 350; Gerstenhaber, M., 238, 350; Gillman, L., 374, 374, 562, 562, 562; Gilmore, P. C., 556; Ginsburg, S., 57, 238, 379, 380, 538; Givens, W., 243; Goffman, C., 538; Goheen, H. E., 169; Goldberg, M., 169; Goldberg, S., 350; Goldberg, S. I., 248, 369, 563; Golomb, M., 68, 68; Gordon, W. L., 62; Gottschalk, W. H., 50; Gough, L., 563; Green, J. W., 83, 538, 539; Green, L. W., 245; Griffin, J. S., Jr., 62, 178, 369; Grosswald, E., 342; Gustin, W., 386; Gutterman, M. M., 362.

Haas, F., 160, 239; Hadlock, E. H., 54; Hailperin, T., 557; Haimo, F., 375; Hall, M., Jr., 375, 385; Halperin, I., 536; Hammer, P. C., 73, 172, 173, 385, 385; Hamstrom, M. E., 178, 563; Handelman, G. H., 361; Harary, F., 179, 387; Harris, T. E., 558; Harrold, O. G., Jr., 369; Hayes, C. A., 452; Heller, I., 551, 551; Helson, H., 160; Henkin, L. A., 84; Henriksen, M., 66, 152, 374, 374, 562, 562, 562, 615; Herriot, J. G., 539; Herstein, I. N., 152; Herzberger, M. J., 539; Herzog, F., 536; Hewitt, E., 179, 397, 539; Higman, D. G., 342; Hind, A. T., Jr., 60; Hocking, J. G., 73, 564; Hodge, P. G., Jr., 82, 455; Hoffman, A. J., 54; Hoggatt, V. E., Jr., 453; Hollcroft, T. R., 526; Holyoke, T. C., 375; Horn, A., 76, 234; Hostinsky, L. A., 343; Hu, S. T., 152; Huber, A., 160, 239; Huff, G. B., 54; Hutcherson, W. R., 556.

Ikenberry, E., 57, 456; Indritz, J., 540; Ingram, W. H., 58; Isaacs, R., 79; Isbell, J. R., 63, 63, 234, 234.

Jackson, J. R., 76, 389, 389; Jacob, H. G., Jr., 235; Jaeger, A., 55, 153; Jardetzky, W. S., 362; Jerison, M., 380, 540, 562, 564; Johnson, R. E., 526; Jones, F. B., 179; J6nsson, B., 77, 77, 343; Juncosa, M. L., 359.

Kadison, R. V., 153, 540; Kalaba, R. E., 247; Kalicki, J., 77; Kalisch, G. K., 527; Kallianpur, G. B., 247, 366; Kaplan, W., 351; Kaplansky, I., 154; Karlin, S., 393; Kasner, E., 173; Katz, L., 564; Kaufman, H., 351; Kazarinoff, N. D., 380; Kelley, J. L., 161, 380; Kemperman, J. H. B., 69, 381; Kiefer, J., 84, 85; Klamkin, M. S., 78, 161, 559; Klee, V. L., Jr., 161, 248, 369; Kleene, S. C., 557; Kleinfeld, E., 154; Koch, R. J., 63; Koehler, F., 381; Kolodner, I. I., 351; Korevaar, J., 381, 540; Kovach, L. D., 78; Kozakiewicz, W., 237, 345; Kravetz, S., 541, 541; Krzywoblocki, M. Z., 382, 552.

Lambek, J., 527, 527, 527; Lane, N. D., 83, 556; Lane, R. E., 453; Lang, S., 41; Langenhop, C. E., 541; Lanzano, P., 173; Lee, T. H., 55; Leger, G. F., Jr., 375; Lehman, R. S., 390; Lehner, J., 394; Leipnik, R. B., 79, 394, 453, 542; Lepson, B., 239; LeVeque, W. J., 154, 154, 528; Levin, E., 455; Levine, J., 61; Levit, R. J., 56; Lewis, D. J., 235, 528; Liao, S. D., 458, 565; Livingston, A. E., 44, 239, 240; Lohwater, A. J., 69; Lorentz, G. G., 161 ; Lotkin, M., 542; Ludford, G. S. S., 362; Lukacs, E., 240, 366; Luke, Y. L., 70; Lyndon, R. C., 235, 340, 343.

McAllister, B. L., 394; McAuley, L. F., 63; McKnight, J. D., Jr., 73; McLaughlin, J. E., 550; McShane, E. J., 560; Mack, S. F., 80; Mackey, G. W., 45; MacLane, G. R., 162; MacNeish, H. F., 47; MacNerney, J. S., 58; Magnus, A., 382; Mann, W. R., 60; Marden, M., 157; Markus, L., 48, 542; Martin, A. V., 397; Marx, I., 244, 550; Massera, J. L., 45; Massey, W. S., 179; Mautner, F. I., 542, 543; Maxfield, J. E., 451; Maxfield, M. W., 451; May, K. O., 70; Medlin, G. W., 56; Michael, E. A., 180, 248, 565; Mientka, W. E., 340; Miles, E. P., 58; Miller, K. S., 395; Minagawa, T., 173; Mirkil, H., 162, 162, 162, 163; Mishoe, L., 537; Moise, E. E., 370, 565; Montgomery, M. D., 565; Moore, C. N., 58, 352; Morawetz, C. S., 352; Mordell, L. J., 528; Mor- 
gan, G. W., 363; Morse, A. P., 453; Moser, L., 527, 527, 527; Mostert, P. S., 74, 387; Mostow, G. D., 51; Motzkin, T. S., 155, 163, 394, 396; Moy, S. T. C., 385; Murdoch, D. C., 528; Murray, G. G., 163.

Nehari, Z., 543; Neumann, B. H., 54; Nirenberg, L., 344; Niven, I., 451; Nomizu, K., 48; Noonan, B., 529; Norman, R. Z., 387; Northam, E. S., 387.

Ohtsuka, M., 453; Osserman, R., 346.

Pan, T. K., 83, 83, 396; Parker, W. V., 56, 56; Parzen, E., 85, 543; Pauc, C. Y., 452; Payne, L. E., 71, 170, 244, 363; Pellicciaro, E. J., 59; Perron, O., 537; Phillips, R. S., 80; Pierce, R. S., 566; Piranian, G., 69, 536; Pitcher, E., 529, 566; Pollard, H., 352; Porcelli, P., 164, 543; Post, E. L., 245, 246, 557; Powell, J. H., 564; Prenowitz, W., 48; Price, C. M., 362; Protter, M. H., 45, 240, 344, 353, 544.

Rabson, G., 380; Rad6, T., 173; Raney, G. N., 41; Ratner, L. T., 544; Redheffer, R. M., 80, 80, 81, 81, 395, 544, 544, 545; Reich, E., 82, 395; Reiner, I., 529; Reza, F. M., 552; Rice, H. G., 557; Rider, P. R., 175; Ritt, R. K., 382, 383, 383; Ritter, I. F., 240; Robbins, H., 50, 247, 366; Roberson, R. E., 81; Roberts, J. H., 370; Robertson, M. S., 164, 241, 545; Robinson, G. de B., 343, 525; Robinson, L., 566; Robinson, L. B., 164, 241, 383; Robinson, L. V., 164, 364; Rogers, H., Jr., 49, 49; Rohde, F. V., 363; Rosenberg, A., 155, 451, 451; Rosenbloom, P. C., 165; Rosenfeld, L., 358; Rosenlicht, M., 241; Rosenthal, A., 69; Roth, J. P., 371; Roth, W. E., 57; Rubin, H., 174; Ruchte, M. F., 529; Rudin, W., 353, 353, 545; Rutledge, W. A., 56.

Saltzer, C., 170, 546; Salzer, H. E., 552, 553; Samelson, H., 177, 567; Samoloff, J., 354; Sanderson, D. E., 567; Sangren, W. C., 67; San Soucie, R. L., 375; Savage, L. J., 397; Schaerf, H. M., 165; Schafer, R. D., 155; Schecter, S., 354; Scheerer, A., 238; Scheid, F., 546; Scherk, P., 83, 556; Schiffer, M. M., 160, 538; Schild, A., 354; Schwartz, H. M., 354, 355; Schwartz, J. T., 165, 165, 174, 546; Schwarz, R. J., 395; Schweitzer, A. R., 174, 175, 175; Schwerdtfeger, H. W. E., 456; Scott, D., 77, 78; Scott, W. R., 376, 530; Seidel, W., 452, 532; Selfridge, R. G., 454; Serrin, J. B., Jr., 170; Shanks, M. E., 74; Shapiro, V. L., 46, 241, 547; Shields, A. L., 59, 547; Shoenfield, J. R., 365; Siegel, K. M., 364; Sion, M., 547; Siry, J. W., 370; Sleator, F. B., 364; Smith, C. B., 60; Smith, K. T., 378, 547; Snyder, H. H., 547, 553; Snyder, W. S., 166, 554; Spencer, D. E., 554; Spencer, G. L., 554, 554; Spitzer, F. L., 176, 176; Sprinkle, H. D., 178; Stalley, R. D., 390; Stark, J. M., 242, 242; Sternberg, H. M., 355; Sternberg, R. L., 351, 355, 364; Stewart, B. M., 376; Stone, W. M., 456; Straus, E. G., 393; Strodt, W., 355, 356, 356, 357; Strother, W. L., 63; Suppes, P. C., 174; Sussman, I., 530; Swift, J. D., 390; Swingle, P. M., 64; Szász, O., 240.

Taam, C. T., 166, 166, 357; Tarski, A., 77, 77, 365, 390; Taussky, O., 54, 57, 155; Taylor, R. L., 40; Thompson, G. L., 530; Thomsen, D. L., Jr., 548; Thorne, C. J., 394; Thrall, R. M., 525; Thron, W. J., 43; Thurston, H. S., 531; van Tijn, D. E., 175; Tintner, G., 559; Titt, E. W., 357; Titus, C. J., 70; Todd, J., 364; Tompkins, C. B., 396; Tompson, R. N., 454; Toralballa, L. V., 180; Turrittin, H. L., 548.

Uhlenbeck, G. E., 179; Ullman, J. L., 166; Utz, W. R., 167, 376.

Vaught, R. L., 391, 396; Vinograde, B., 376.

Wahab, J. H., 57; Wall, H. S., 167; Wallace, A. D., 531, 567, 568; Walsh, J. L., 163, 242, 358, 358, 394; Walsh, M. J., 64; Wang, C. Y., 358; Wang, H., 557, 558; Wang, H. C., 64, 180; Ward, L. E., Jr., 180; Wasow, W. R., 83; Weinberger, H. F., 43, 244, 346, 363; Weiner, L. M., 155, 377; Wendel, J. G., 548; Weinstein, A., 359, 454; Wermer, J., 243, 243; Wertheim, D. G., 161; Whaples, G., 66, 156, 377; Whiteman, A. L., 343; Widder, D. V., 170; Wilansky, A., 243; Wilder, R. L., 371; Willcox, A. B., 235; Williams, R. F., 458; Wolf, F., 359; Wolfe, J. H., 177; Wolfowitz, J., 84, 85, 85, 457, 457; Wolfson, K. G., 236, 344, 359; Wollan, G. N., 60; Wright, F. B., 531, 549; Wyler, O., 71, 174.

Yamabe, H., 371; Yang, C. T., 568; Yood, B., 46, 46; Young, D. M., Jr., 47, 242, 359; Young, G. S., 70. 
Zarantonello, E. H., 244; Zassenhaus, H. J., 41; Zelinsky, D., 451; Ziebur, A. D., 549; Zimmerberg, H. J., 46; Zygmund, A., 452, 534.

\section{INDEX OF REPORTS OF MEETINGS AND MISCELLANEOUS ARTICLES}

Bridgeman, P. W., Quine, W. V., Van Vleck, J. H., and Widder, D. V. Edward Vermilye Huntington, 1874-1952, 399.

Chatland, H. See Green, J. W.

Cohen, L. W. Reports of meetings of the American Mathematical Society: October Meeting in Nerw Haven, 39; February Meeting in New York, 231; April Meeting in New York, 330; Summer Meeting in Kingston, 515.

Duren, W. L. The support of mathematical research by the National Science Foundation, 1.

Green, J. W. Reports of meetings of the American Mathematical Society: November Meeting in Los Angeles, 75; May Meeting in Palo Alto, 388.

Green, J. W., and Chatland, H. Reports of meetings of the American Mathematical Society: June Meeting in Missoula, 449.

Green, J. W., and Lehmer, D. H. Reports of meetings of the American Mathematical Society: Sixth Symposium in Applied Mathematics, 513.

Huntington, E. V. See Bridgman, P. W.

Lehmer, D. H. See Green, J. W.

Meder, A. E., Jr. A communication from the treasurer, 459.

Quine, W. V. See Bridgman, P. W.

Steenrod, N. E. The conference on fiber bundles and differential geometry in Ithaca, 569.

Van Vleck, J. H. See Bridgman, P. W.

Whyburn, W. M. Reports of meetings of the American Mathematical Society: November Meeting in Raleigh, 52.

Widder, D. V. See Bridgman, P. W.

Youngs, J. W. T. Reports of meetings of the American Mathematical Society: November Meeting in Lafayette, 65; Annual Meeting in St. Louis, 140; April Meeting in Chicago, 372.

\section{INDEX OF BOOK REVIEWS}

Ackermann, W. See Hilbert, D.

Ahlfors, L. V. Complex analysis. A. C. Schaeffer, 464.

Artin, E. See Bourbaki, N.

Baer, R. Linear algebra and projective geometry. I. Kaplansky, 400.

Bagemihl, F. See Borel, E., Novozhilov, V. V., Pontryagin, L. S.

Bateman, P. T. See Davenport, H.

Beckenbach, E. F. See Construction and applications of conformal maps.

Bessel functions. Part II. Functions of positive integer order. Ed. by W. G. Bickley, L. J. Comrie, J. C. P. Miller, D. H. Sadler, and A. J. Thompson. A. Erdélyi, 189.

Bianchi, L. Opere. 416.

Bickley, W. G. See Bessel functions.

Bing, R. H. See Sierpinski, W.

Boas, R. P. See Journal of Rational Mechanics and Analysis, Michigan Mathematical Journal.

Borel, E. Les nombres inaccessibles. F. Bagemihl, 406.

Bourbaki, N. Intégration. P. R. Halmos, 249.

Bourbaki, N. Éléments de mathêmatique. Book II. Algebra. Chaps. I-VII. E. Artin, 474.

Burkill, J. C. The Lebesgue integral. L. H. Loomis, 89.

Chern, S. S. See Wu, W. T.

Chevalley, C. See Hasse, H.

Collatz, L. Numerische Behandlung von Differentialgleichungen. W. E. Milne 94. 
Comrie, L. J. See Bessel functions.

Construction and applications of conformal maps. Ed. by E. F. Beckenbach. 484.

Curry, H. B. See Hilbert, D.

Davenport, H. The higher arithmetic. P. T. Bateman, 473.

Day, M. M. See Hamburger, H. L.

DeCicco, J. See Sokolnikoff, I. S.

Dedekind, R. See Riemann, B.

Deuxième Colloque de Géométrie Algêbrique. M. Rosenlicht, 415.

Diaz, J. B. See Pólya, G.

Dieudonné, J. See Jacobson, N., Mayrhofer, K.

Dilworth, R. P. See Rennie, B. C.

DuVal, P. See Semple, J. G.

Einstein, A. The meaning of relativity. 485.

Erdélyi, A. See Bessel functions.

Estermann, T. Introduction to modern prime number theory. L. Schoenfeld, 255.

Fluid dynamics, 485.

Fraenkel, A. A. Abstracts set theory. P. R. Halmos, 584.

Frink, O. See Robinson, A., Wilder, R. L.

Fuchs, W. H. J. See Mandelbrojt, S.

Godeaux, L. Les transformations birationnelles du plan, 485.

Goffman, C. Real functions. M. E. Munroe, 572.

Goodman, A. W. See Thron, W. J.

Grimshaw, M. E. See Hamburger, H. L.

Halmos, P. R. Measure theory. J. C. Oxtoby, 89.

Halmos, P. R. See Bourbaki, N., Fraenkel, A. A., Picone, M.

Hamburger, H. L., and Grimshaw, M. E. Linear transformations in n-dimensional vector space. An introduction to the theory of Hilbert space. M. M. Day, 98.

Hardy, G. H., Littlewood, J. E., and Polya, G. Inequalities. A. Zygmund, 411.

Hasse, H. Über die Klassezahl abelscher Zahlkörper. C. Chevalley, 281.

Hilbert, D., and Ackermann, W. Grundzige der theoretischen Logik. H. B. Curry, 263.

Hilbert, D., and Ackermann, W. Principles of mathematical logic. Trans. by G. G. Leckie and F. Steinhardt. Ed. by R. E. Luce. H. B. Curry, 263.

Hodge, W. V. D. The theory and applications of harmonic integrals, 415.

Jacobson, N. Lectures in abstract algebra. Vol. II. Linear algebra. J. Dieudonne, 480.

Journal of Rational Mechanics and Analysis. R. P. Boas, 191.

Kaplansky. I. See Baer, R.

Kneebone, G. T. See Semple, J. G.

Komm, H. See Novozhilov, V. V., Pontryagin, L. S.

Krieger, C. C. See Sierpinski, W.

Kuratowski, C. Topologie. Vol. I. 484.

Lappo-Danilevsky, J. A. Mémoires sur la theorie des systèmes des équations differentielles linéaires, 485.

Leckie, G. G. See Hilbert, D.

Levinson, N. See Sauer, R.

Lewy, H. See Riemann, B.

Littlewood, D. E. A university algebra. D. Zelinsky, 97.

Littlewood, J. E. See Hardy, G. H.

Loomis, L. H. See Burkill, J. C.

Lorch, E. R. See Riesz, F.

Luce, R. E. See Hilbert, D.

McKinsey, J. C. C. Introduction to the theory of games. J. Wolfowitz, 267.

McNaughton, R. See Tarski, A. 
Mandelbrojt, S. Séries adherentes. Régularisation des suites. Applications. W. H. J. Fuchs, 413.

Massey, W. S. See Pontryagin, L. S.

Mayrhofer, K. Inhalt und Mass. J. Dieudonné, 479.

Michigan Mathematical Journal. R. P. Boas, 483.

Miller, J. C. P. See Bessel functions.

Milne, W. E. Numerical solution of differential equations. D. Young, 577.

Milne, W. E. See Collatz, L.

Munroe, M. E. See Goffman, C., Rudin, W., Thielman, H. P.

Murray, F. J. See Willers, F. A.

Neyman, J. See Proceedings of the Second Berkeley Symposium on Mathematical Statistics and Probability.

Noether, M. See Riemann, B.

Novozhilov, V. V. Foundations of the nonlinear theory of elasticity. Trans. by F. Bagemihl, H. Komm, and W. Seidel. C. Truesdell, 467.

Oxtoby, J. C. See Halmos, P. R.

Parodi, M. Sur quelques propriétés des valeurs caractéristiques des matrices carrées. O. Taussky, 464.

Picone, M., and Viola, T. Lezioni sulla teoria moderna dell'integrazione. P. R. Halmos, 94.

Pólya, G. See Hardy, G. H.

Pólya, G., and Szegö, G. Isoperimetric inequalities in mathematical physics. J. B. Diaz, 588.

Pontryagin, L. S. Foundations of combinatorial topology. Trans. by F. Bagemihl, H. Komm, and W. Seidel. W. S. Massey, 188.

Proceedings of the Second Berkeley Symposium on Mathematical Statistics and Probability. Ed. by J. Neyman. 192.

Reeb, G. See Wu, W. T.

Reissner, E. See Roy, M.

Rennie, B. C. The theory of lattices. R. P. Dilworth, 100.

Riemann, B. The collected works of Bernhard Riemann. Ed. by H. Weber, with the assistance of R. Dedekind. With a supplement ed. by M. Noether and W. Wirtinger, and an introduction by $\mathrm{H}$. Lewy, 484 .

Riesz, F., and Sz.-Nagy, B. Leçons d'analyse fonctionelle. E. R. Lorch, 270.

Robinson, A. On the metamathematics of algebra. O. Frink, 582.

Rosenlicht, M. See Deuxième Colloque de Géométrie Algébrique.

Roy, M. Mécanique des milieux continus et deformables. E. Reissner, 99.

Rudin, W. Principles of mathematical analysis. M. E. Munroe, 572.

Sadler, D. H. See Bessel functions.

Sauer, R. Anfangswertprobleme bei partiellen Differentialgleichungen. N. Levinson, 410.

Schaeffer, A. C. See Ahlfors, L. V.

Schoenfeld, L. See Estermann, T.

Seidel, W. See Novozhilov, V. V., Pontryagin, L. S.

Semple, J. G., and Kneebone, G. T. Algebraic projective geometry. P. DuVal, 571.

Sibagaki, W. Theory and application of the gamma function, with a table of the gamma function for complex arguments significant to the sixth decimal place, 485.

Sierpinski, W. General topology. Trans. by C. C. Krieger. R. H. Bing, 410.

Smiley, M. F. See Stoll, R. R.

Sokolnikoff, I. S. Tensor analysis. Theory and applications. J. DeCicco, 86.

Steinhardt, F. See Hilbert, D.

Stoll, R. R. Linear algebra and matrix theory. M. F. Smiley, 96.

Synge, J. L. See Weinstock, R. 
Szegö, G. See Polya, G.

Sz.-Nagy, B. See Riesz, F.

Tables of Chebyshev polynomials $S_{n}(x)$ and $C_{n}(x), 484$.

Tarski, A. A decision method for elementary algebra and geometry. R. McNaughton, 91.

Taussky, O. See Parodi, M.

Thielman, H. P. Theory of functions of real variables. M. E. Munroe, 572.

Thompson, A. J. See Bessel functions.

Thron, W. J. Introduction to the theory of functions of a complex variable. A. W. Goodman, 585.

Truesdell, C. See Novozhilov, V. V., Westergaard, H. M.

Viola, T. See Picone, M.

Weber, H. See Riemann, B.

Weinstock, R. Calculus of variations with applications to physics and engineering. J. L. Synge, 402.

Westergaard, H. M. Theory of elasticity and plasticity. C. Truesdell, 412.

Wilder, R. L. Introduction to the foundations of mathematics. O. Frink, 580.

Willers, F. A. Mathematische Maschinen und Instrumente. F. J. Murray, 100.

Wirtinger, W. See Riemann, B.

Wolfowitz, J. See McKinsey, J. C. C.

$\mathrm{Wu}, \mathrm{W}$. T., and Reeb, G. Sur les espaces fibrés et les varietés feuilletées. S. S. Chern, 258.

Young, D. See Milne, W. E.

Zelinsky, D. See Littlewood, D. E.

Zygmund, A. See Hardy, G. H.

Aronszajn, N., 65.

\section{INDEX OF INVITED ADDRESSES ${ }^{1}$}

Artin, E., 332.

Bochner, S., 516.

Cartan, H., 516.

Chow, W. L., 232.

Dieudonné, J. A., 372.

Recent developments in the theory of locally convex vector spaces, 495.

Forsythe, G. E. Solving linear algebraic equations can be interesting, 299.

Givens, W., 52.

Gleason, A. M., 141.

Goldstine, H. H., 52.

Hildebrandt, T. H. Integration in abstract spaces, 111.

Leray, J., 39.

Loève, M. M., 75.

Lonseth, A. T., 449.

Massey, W. S., 331.

Morse, M., 141.

von Neumann, J., 141.

Rothe, E. H. Gradient mappings, 5.

Schoenberg, I. J. On smoothing operations and their generating functions, 199.

Snapper, E., 388.

Weinstein, A. Generalized axially symmetric potential theory, 20.

Wilder, R. L., 141.

- The origin and growth of mathematical concepts, 423 .

Zygmund, A., 516.

${ }^{1}$ Where the title of the address is not given, the reference is to the Report of the Meeting at which the address was given. 